\title{
Assessment of the Effectiveness of Hand Therapy for Elderly People with Dementia
}

\author{
Hiromi Toki ${ }^{1,2}{ }^{*}$, Hiroko Kunikata1, Yurie Fujiwara², Shin Fujisawa ${ }^{2}$ \\ ${ }^{1}$ Department of Nursing, Faculty of Health Sciences, Kagawa Prefectural University of Health Sciences, Kagawa, Japan \\ ${ }^{2}$ Iwaki Hospital, Iwakikai Medical Corporation, Kagawa, Japan \\ Email: ^toki@chs.pref.kagawa.jp
}

How to cite this paper: Toki, H., Kunikata, H., Fujiwara, Y. and Fujisawa, S. (2022) Assessment of the Effectiveness of Hand Therapy for Elderly People with Dementia. Open Journal of Therapy and Rehabilitation, 10, 25-37.

https://doi.org/10.4236/ojtr.2022.101003

Received: November 27, 2021

Accepted: January 17, 2022

Published: January 20, 2022

Copyright (c) 2022 by author(s) and Scientific Research Publishing Inc. This work is licensed under the Creative Commons Attribution International License (CC BY 4.0).

http://creativecommons.org/licenses/by/4.0/

\begin{abstract}
Background: This study evaluated the effectiveness of hand therapy for elderly people with dementia and the change in effectiveness depending on sex differences between the patients and operators. Methods: The Abe's behavioral and psychological symptoms of dementia score (ABS), index of resistance to care, and inner balance scanner scores were evaluated. Results: The mean ABS was 18.4 (before therapy), 16.0 (operator of the same sex), and 17.1 (operator of the opposite sex). The mean index scores for resistance to care were 15.3 (before therapy), 15.2 (operator of the same sex), and 13.2 (operator of the opposite sex). When the operator and patient were of the same sex, the mean value of comparison of total power (TP) decreased from 28,060.4 (before therapy) to 5974.3 (after therapy), and the mean low frequency (LF)/high frequency (HF) ratio decreased from 1.7 (before therapy) to 1.2 (after therapy). When the operator was of the opposite sex of that of the patient, the mean TP value decreased from 25,760.4 (before therapy) to 7669.5 (after therapy), and the mean LF/HF ratio increased from 1.2 (before therapy) to 1.5 (after therapy). Conclusions: Hand therapy was the most effective when the patient and operator were of the same sex.
\end{abstract}

\section{Keywords}

Older Adults, Cognitive Impairment, Massage Therapy, Case Report

\section{Introduction}

Currently, dementia is treated by providing drug therapy and various non-drug therapies. Rehabilitation and psychotherapy, which are non-drug therapies, not only enhance the power of people with dementia, but also maximize their ability to function well. Furthermore, supporting the family and preparing a good environment contributes to psychological stability. 
In addition to cardinal symptoms of dementia, such as memory loss and cognitive impairment, behavioral and psychological symptoms of dementia (BPSD), including hallucinations, delusions, wandering, and violence, are common. BPSD are involved in the progression of dementia and dementia-related deaths [1] and reduce the quality of life (QOL) of elderly people with dementia [2] [3]. When dementia progresses from a mild to moderate stage, BPSD commonly occur, causing various problems, such as difficulty in home caring, reductions in caregiver and patient QOL, and increase in caregiver stress [4] [5]. Therefore, the management of BPSD is of particular importance. Even according to the Japan's Ministry of Health, the principle of non-drug therapy is the first choice for BPSD [6]. Administration of prescription drugs to elderly people with dementia bears risks including falls and can cause adverse effects [7] [8]. Therefore, a non-pharmacological therapeutic approach is recommended. A review reported that non-drug interventions, such as aromatherapy and relaxation, are effective in improving BPSD [9].

Although the practical application of hand therapy is recommended for elderly people with dementia, there are few reports that are mainly subjective evaluations [10] [11] [12] [13]. Furthermore, most of the studies concern healthy people, and only a few reports on people with health problems exist. The diverse effects of hand therapy, as reported from subjective evaluations, include increased comfort, [14] [15] reduced anxiety, [16] [17] [18] alleviated rash pain, [19] [20] increased familiarity with the practitioner, [21] feeling of relaxation, [17] [22] and decreased feelings of anger, hostility, and fatigue [17]. Additionally, the effect of hand care on autonomic nervous system function is reported from a significant decrease in heart rate [17] [23]. Especially, hand therapy stimulates the tactile nerves through touching, which brings calmness and a sense of security and creates a sense of intimacy and trust between the caregiver and the patient.

However, in clinical practice, elderly people with dementia and BPSD may refuse to be touched. We have a notion that same-sex nurses experience less resistance to care. Therefore, in our opinion, receiving hand therapy from same-sex nurses might be even more effective for elderly people with dementia who experience $\mathrm{BPSD}$.

Accordingly, this study aimed to assess the effectiveness of hand therapy in elderly patients with dementia and the effect of sex differences between patients and operators to provide suggestions for the nursing care of dementia. To study these objectives, the Abe's behavioral and psychological symptoms of dementia score (ABS) [24] and index of resistance to care (prepared by nurses) were calculated, and the autonomic nervous system function was measured using the inner balance scanner (IBS).

\section{Methods}

\subsection{Subjects}

The study included three elderly patients who were hospitalized in a dementia 
ward. The study protocol is shown in Table 1 . All patients exhibited resistance toward the staff regarding toileting (diaper changing/leading to the toilet) and bathing issues. Regarding medication, patient $\mathrm{A}$ was prescribed quetiapine tablets (Quetiapine fumarate); patient B was prescribed a yokukansanryo extract (Yokukansan); and patient $\mathrm{C}$ was prescribed Belsomra (Suvorexant), Menesit (Levodopa), and Effexor SR capsules (Venlafaxine hydrochloride). All drugs have been shown to influence BPSD and resistance to care; the prescriptions remained unchanged during the study period.

\subsection{Procedure}

Data were collected over a total of 20 days between October and November 2018. Measurements were taken at four time points during the following 5-day periods: before hand therapy, during hand therapy by a male operator, during a rest period, and during hand therapy by a female operator (Table 2 ).

\subsection{Protocol for Hand Therapy}

Hand therapy is characterized by slow and light rubbing (patting) in such a way that the body is softly wrapped [25]. Hand therapy was performed before bathing (at approximately $9 \mathrm{am}$ ) at the subject's bedside while sitting in a chair. A humidity level at which the subjects felt comfortable was maintained, and the measurements were taken in a quiet environment lasting 5 minutes for each hand. The protocol of hand therapy is the following.

Table 1. Profile of the three patients.

\begin{tabular}{ccccc}
\hline Subjects & Sex & Age & Disease & MMSE score \\
\hline A & F & early 90s & AD & 3 \\
B & F & late 80s & AD & 1 \\
C & M & early 70s & AD & 10 \\
\hline
\end{tabular}

AD, Alzheimer's disease; MMSE, Mini Mental State Examination.

Table 2. Research protocol.

\begin{tabular}{|c|c|c|c|c|}
\hline & $\begin{array}{c}\text { Pre-therapy } \\
\text { period }\end{array}$ & $\begin{array}{l}\text { Hand therapy by } \\
\text { a male operator }\end{array}$ & Rest & $\begin{array}{c}\text { Hand therapy } \\
\text { by a female } \\
\text { operator }\end{array}$ \\
\hline Abe's BPSD score & 5 days & 5 days & 5 days & 5 days \\
\hline Index of resistance to care & 5 days & 5 days & 5 days & 5 days \\
\hline Inner Balance Scanner & - & Day $1,3,5$ & - & Day $1,3,5$ \\
\hline
\end{tabular}

Abe's BPSD score: daily assessments of average conditions by the nurse-in-charge, who worked from $9 \mathrm{AM}$ to $4 \mathrm{PM}$. Index of resistance to care: daily assessments of average conditions by the respective nurses-in-charge, who worked day and night shifts. Inner balance scanner: measurements before and after hand therapy on days 1, 3, and 5 of each 5-day therapy course. 
1) First, explain that you will be doing hand therapy and get consent. And the operators sat at a distance that allowed their knees to maintain contact with the patient's body, and the therapy began by wrapping both hands of the patient with a towel.

2) Oil was poured on the patient's palm and spread, covering the entire area of the hands.

3) The oil was spread from the wrist toward the fingertip such that the operator's and patient's hands were not separated.

4) The palms were rubbed from the center, moving outward (right/left) with the pulp of the thumb.

5) The area between the bones on the back of the hand from the wrist toward the fingertip was rubbed.

6) Each finger was rubbed carefully, one by one, in the order of side, back/palm sides, and the whole finger.

7) To conclude the care on the back of the hand, the wrist to fingertips were rubbed.

8) The hands were flipped while wrapped with both the operator's hands.

9) The palms were rubbed from the center toward the outside (right/left) and from the wrist toward the fingertip.

10) The palms were rubbed with three fingers in a circular motion.

11) Slowly and carefully, the palms were rubbed from the wrist to the fingertip with the operator's palm.

12) After finishing the palm, the patient's hands were flipped while wrapping with both hands.

13) The back of the wrists, from the center moving outward (right/left), were rubbed.

14) After softly wrapping with both hands of the operator, the patient's hands were wrapped with a towel.

\subsection{Measurement Tools and Analyses}

\section{ABS}

The ABS [24] is a simple self-administered BPSD score for dementia caregivers. Each of the 10 BPSD items observed in patients with dementia are allotted a score $(0$ - 9) based on the frequency and severity of symptoms, with the degree of BPSD determined by the total score. A score of 44 indicates the highest severity of BPSD. An average score for each period was calculated as follows: before hand therapy, during hand therapy by a male operator, during a rest period, and during hand therapy by a female operator. Additionally, an average was calculated for each category as follows: before hand therapy, when the operator and the patient were of the same sex, and when the sex of the operator was different from that of the patient. In the assessment of BPSD with the ABS, average conditions between 9 am and 4 pm were assessed by a day shift nurse-in-charge every day for a period of 20 days in which a male and a female operator performed hand therapy. To achieve accuracy/reliability, we had an explanatory meeting 
with the nursing staff before data collection.

\section{Index of resistance to care}

Resistance to care is defined as conditions in which a patient refuses to receive care by a caregiver despite caregiving being needed. In this study, the term specifically represents strong resistance to care regarding toileting/bathing.

Four items of resistance to care were extracted by caregivers for each patient (Table 3). An average for each period was calculated as follows: before hand therapy, during hand therapy by a male operator, during a rest period, and during hand therapy by a female operator. Additionally, an average was calculated for each category as follows: before hand therapy, when the operator and the patient were of the same sex, and when the sex of the operator was different from that of the patient. The average conditions were assessed with a score of 1 (weak) to 3 (strong) by a day and a night shift nurse-in-charge for a period of 20 days during which a male and a female operator performed hand therapy.

\section{IBS}

The IBS (BIOCON Technologies) is a device that measures changes in time intervals between heartbeats. This is performed using a sensor placed on the earlobe and serves as an indicator of autonomic nervous system function. This study focused on the comparisons of total power (TP), low frequency (LF)/high frequency (HF) ratio, and balance between the sympathetic and parasympathetic nervous systems. TP represents the total power of power spectra of frequencies (very low frequency(VLF), LF, and HF) in a 5-minute test. TP reflects the activity of the autonomic nervous system, which mainly consists of sympathetic nerve activity. TP is calculated by the square of $1 / 1000$ second. LF/HF is a power ratio that represents the overall balance between sympathetic and parasympathetic nervous activities; a high value indicates dominance of sympathetic nervous activity, while a low value indicates dominance of parasympathetic nervous activity.

The measurement of the balance of the autonomic nervous system with the IBS was performed on three days during each period of hand therapy by a male or a female operator (Table 2). The measurement lasted 5 minutes. The patients sat in a chair at the bedside, and the measurement was performed in a quiet environment. An average was calculated for each category: before hand therapy, when the operator and the patient were of the same sex, and when the sex of the operator was different from that of the patient.

\subsection{Ethical Considerations}

The study was conducted in accordance with the World Medical Association's

Table 3. Items of resistance to care in each patient.

\begin{tabular}{lllll}
\hline Subjects & & & \\
A & Spitting & Biting & Violent language/Angry roar & Pinching \\
B & Brushing away & Hitting & Shouting/Angry roar & Touching clothes \\
C & Clasping hands Biting & Violent language/Angry roar & Brushing away \\
\hline
\end{tabular}


Helsinki Declaration for Human Studies. Before conducting the study, we orally explained the objectives and methods of the study, as well as the advantages and disadvantages related to participation in the study to the patients and their families in a written form. We also ensured that participation in the study could be terminated at any time during the study period, and that privacy is strictly protected. Consent was obtained from all participants. Patient data were classified using a personal number to ensure anonymity and were strictly managed. The study was approved by the Ethical Review Committee for Nursing Studies, Committee of Iwaki Hospital, Iwakikai Medical Corporation (No. 18-1).

\section{Results}

\subsection{ABS}

The ABS was lower during the period of rest and during therapy with a female operator than before therapy and during therapy with a male operator (Figure 1). The mean ABS for patient A was 22.0 (before therapy), 24.8 (therapy by a male operator), 12.8 (during rest), and 12.4 (therapy by a female operator). That for patient B was 14.6 (before therapy), 15 (therapy by a male operator), 8.2 (during a rest), and 10.4 (therapy by a female operator). Finally, the ABS for patient $\mathrm{C}$

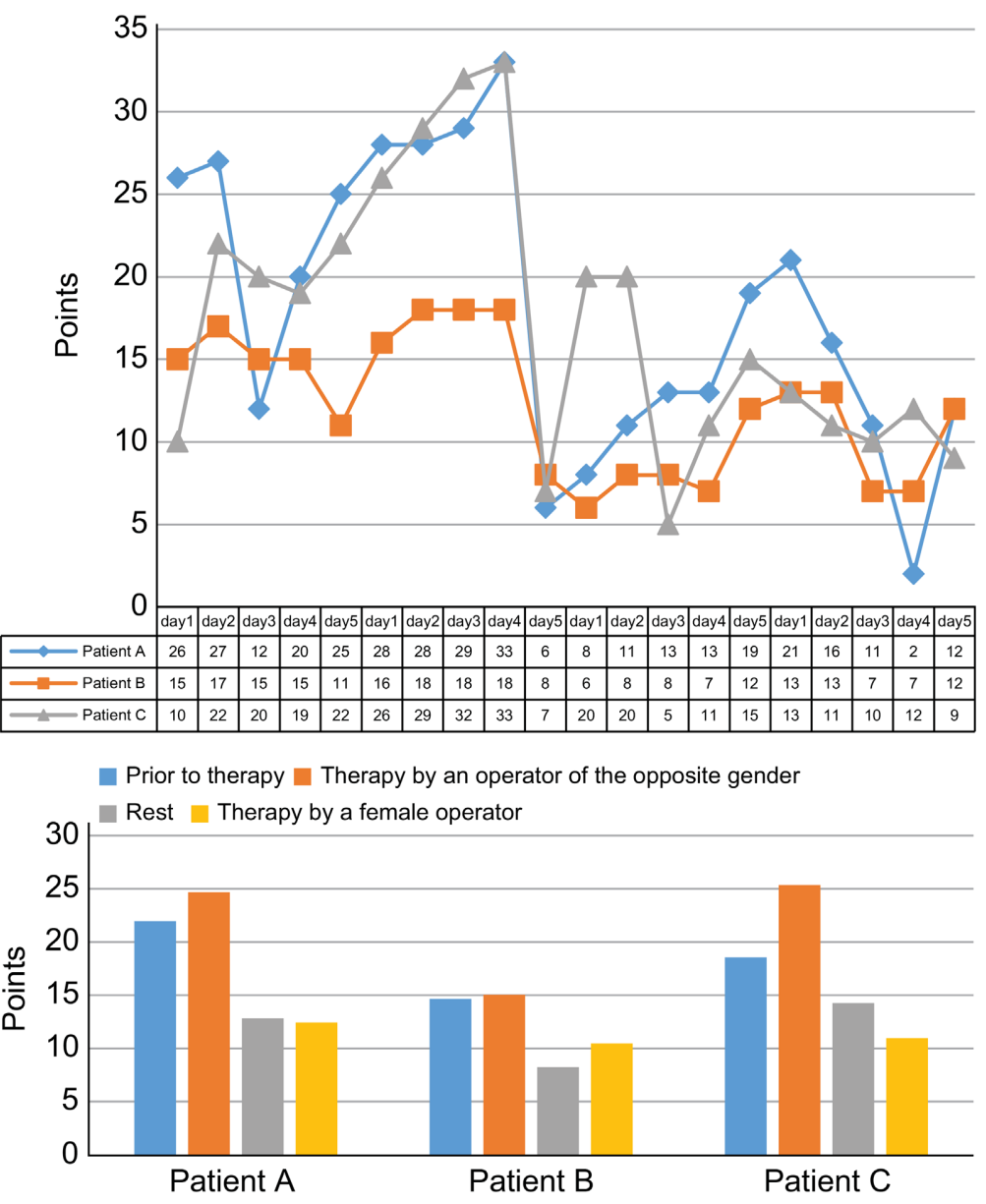

Figure 1. Changes in the ABS. 
was 18.6 (before therapy), 25.4 (therapy by a male operator), 14.2 (during rest), and 11 (therapy by a female operator).

\subsection{Index of Resistance to Care}

Figure 2 shows changes in the patients' levels of resistance to care following hand therapy. Less variability between the different conditions was observed based on mean index scores as compared with the ABS. The mean index scores for patient A were 12.4 (before therapy), 11.4 (therapy by a male operator), 13.2 (during rest), and 12.0 (therapy by a female operator). The scores for patient B were 16.8 (before therapy), 15.8 (therapy by a male operator), 15.6 (during rest), and 15.8 (therapy by a female operator).

The mean index scores for patient $\mathrm{C}$ were 16.6 (before therapy), 17.8 (therapy by a male operator), 14.2 (during rest), and 12.6 (therapy by a female operator).

\subsection{Mean ABS/Index Scores of Resistance to Care by Sex Difference}

Changes in the ABS and resistance to care due to hand therapy performed by an operator of the opposite sex in the three patients are shown in Figure 3. The mean ABS was 18.4 (before therapy), 16.0 (therapy by an operator of the same

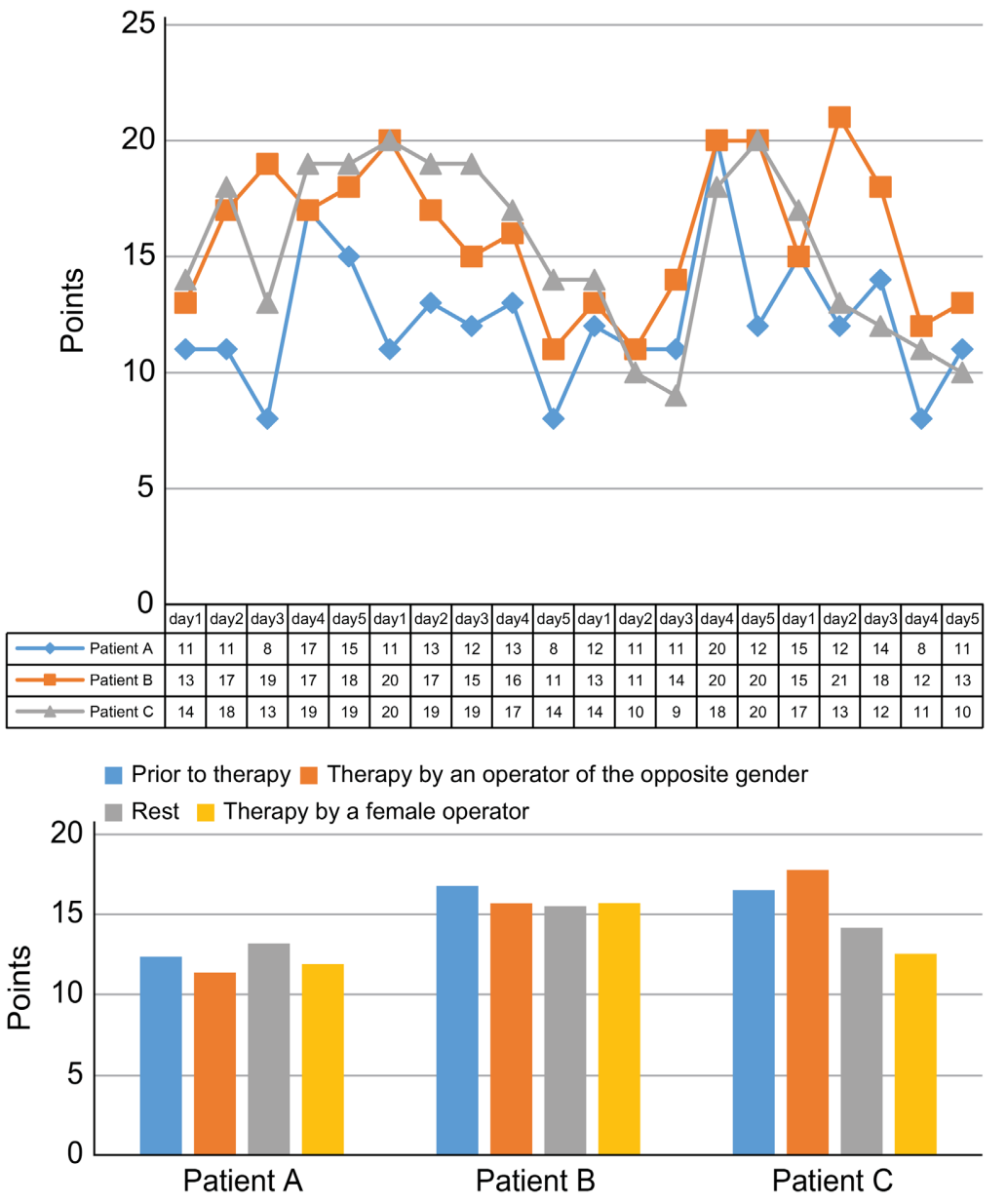

Figure 2. Changes in the patients' levels of resistance. 


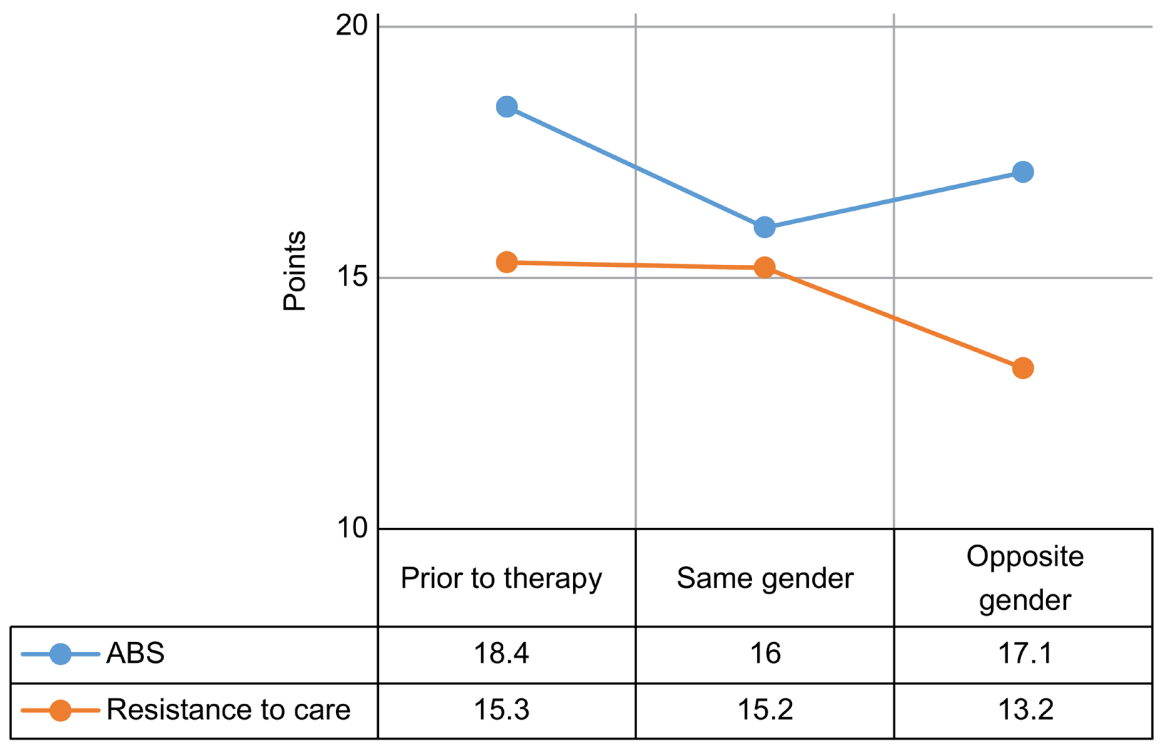

Figure 3. Mean ABS/index scores of resistance to care by sex difference between the patient and the operator.

sex), and 17.1 (therapy by an operator of the opposite sex). The mean index scores of resistance to care were 15.3 (before therapy), 15.2 (therapy by an operator of the same sex), and 13.2 (therapy by an operator of the opposite sex). The ABS was low with a same-sex operator, and the index scores of resistance to care were low during therapy by an operator of the opposite sex. Both the ABS and index scores of resistance to care were lower than those observed before therapy.

\subsection{IBS: Changes in Mean Values among the Three Patients}

The mean TP and LF/HF values calculated by IBS of the three patients are shown in Figure 4. When the operator and the patient were of the same sex, the mean TP values decreased from 28,060.4 (before therapy) to 5974.3 (after therapy), and the mean LF/HF values decreased from 1.7 (before therapy) to 1.2 (after therapy).

When the sex of the operator was different from that of the patient, the mean TP values decreased from 25,760.4 (before therapy) to 7669.5 (after therapy), and the mean LF/HF values increased from 1.2 (before therapy) to 1.5 (after therapy). In the cases of same-sex and opposite-sex operators, the mean TP values decreased, which indicates that sympathetic nervous activity decreased. As the mean LF/HF values decreased when the sex of the operator was the same as that of the patient, parasympathetic nervous activity was dominant, while sympathetic nervous activity was dominant when the operator was of the opposite sex, as the mean $\mathrm{LF} / \mathrm{HF}$ values increased.

\section{Discussion}

The number of subjects in this study was small, and the duration of this study was limited. However, this study found that hand therapy may serve as a 

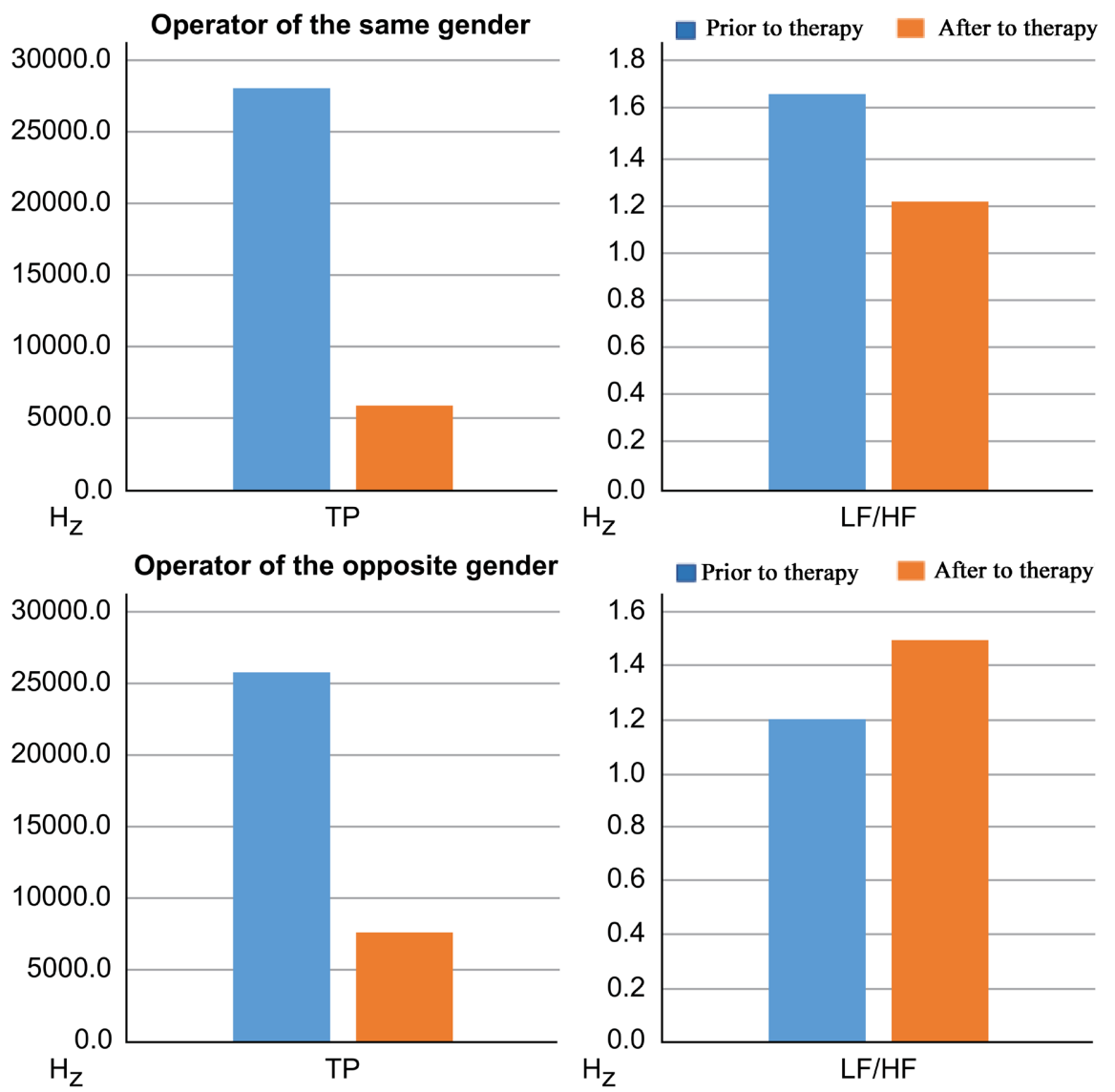

Figure 4. Changes in the mean TP and LF/HF values in IBS among the three patients.

non-pharmacological strategy to improve behavioral and psychological symptoms in people with dementia. Additionally, the effects of sex difference between the patient and the operator have been highlighted, proposing the application of this knowledge for long-term care. However, further research is still needed to provide definite recommendations regarding frequency and types of hand therapy [26].

In all patients, the mean $\mathrm{ABS}$ increased during the first hand therapy session performed by a male operator. However, following a 5-day period of rest, the mean ABS was observed to be lower than that before the hand therapy.

The mean ABS further declined during the second hand therapy session performed by a female operator in patients A and C. We found a decrease in the index scores of resistance to care during hand therapy in patient $\mathrm{A}$. The scores also decreased during hand therapy in patient $\mathrm{B}$ and were maintained during a period of rest. In patient $C$, when hand therapy began, the index scores of resistance to care increased; however, they decreased on continuation of therapy. Regarding autonomic nervous system activity, the sympathetic nervous activity decreased following hand therapy, and a relaxing effect was observed in all the patients. Suzuki reported that reduction in anxiety and behavioral stabilization can be expected in elderly people with dementia by performing tactile care [25]. Accordingly, our study demonstrated that performing hand therapy continually 
reduces $\mathrm{BPSD}$ and resistance to care and confers a calming effect. The study also showed that the effectiveness of hand therapy varies depending on individuals; however, the therapy is expected to become effective when performed continually.

The analysis of changes due to the sex of the operators showed that the therapy was the most effective for reducing BPSD when patients underwent hand therapy by an operator of the same sex. Additionally, the therapy was the most effective for reducing resistance to care when patients underwent hand therapy by an operator of the opposite sex. However, two out of the three patients were women, and a female operator performed the second hand therapy session, which may have moderately influenced the results. The results for the IBS measurements showed that in the case of an operator of the same sex, the parasympathetic nervous activity is dominant. Thus, the present study demonstrated that hand therapy is effective for reducing BPSD; the relaxation effect is elevated when the sex of the operator is the same as that of the patient, whereas when the operator is of the opposite sex, the therapy is effective for reducing resistance to care.

At the initiation of hand therapy, BPSD exacerbated temporarily, presumably owing to the anxiety and fear of being touched by other people due to the decreased cognitive function of the patients. Therefore, when touching elderly people with dementia, given their suspected anxiety and fear of being touched, caregivers should attempt to induce a feeling of security. Continual hand therapy allows for reduction in BPSD and resistance to care as well as an increase in relaxation. Thus, a feeling of security can further result in favorable outcomes.

Concerning the influence of the sex of the operators, resistance to care was shown to decrease with operators of the opposite sex. Although the male patient in this study fiercely resisted care given especially by female staff, resistance to care was reduced by undergoing hand therapy. Thus, being touched by a person of the opposite sex may be recognized not as an uncomfortable experience invoking fear and anxiety, but an experience of obtaining a sense of security by undergoing hand therapy. As BPSD were alleviated, and the hand therapy was more effective for relaxation when performed by an operator of the same sex, elderly people with dementia were considered to obtain a higher sense of security when being touched by a person of the same sex. Therefore, it is necessary to provide nursing care that respects the patient's sex, considering that sex differences affect common resistance to nursing care such as toileting and bathing habits.

Currently, there is little evidence regarding non-pharmacological management for BPSD [27]. Therefore, it is important to evaluate various non-pharmacological approaches using a scale that can accurately evaluate their therapeutic effects. The results of this study provide new insights to the use of hand therapy as a therapeutic treatment for BPSD. However, as the sample size of this study was small, larger studies in the future are needed to confirm the reliability of our results. 


\section{Conclusion}

This study showed that hand therapy is effective for the improvement of BPSD and resistance to care and provides relaxation. In elderly people with dementia, the ABS was reduced when undergoing hand therapy performed by an operator of the same sex, and thus a higher relaxation effect can be expected. Additionally, undergoing hand therapy by an operator of the opposite sex allows patients to effectively reduce resistance to care.

\section{Acknowledgements}

The authors would like to express their heartfelt thanks to the three subjects and their families for their understanding and cooperation with this research. Moreover, the authors would like to acknowledge the assistance of Dr. Tomoyuki Watanabe and the Director of Nursing Tomie Kusaka of Iwaki Hospital (Kagawa, Japan).

\section{Conflicts of Interest}

The authors declare no conflicts of interest regarding the publication of this paper.

\section{References}

[1] Matthew, E.P., Sarah, S., Dingfen, H., Peter, V.R., Martin, S., Joann, T.T. and Constantine, G.L. (2015) Neuropsychiatric Symptoms as Predictors of Progression to Severe Alzheimer's Dementia and Death: The Cache County Dementia Progression Study. American Journal of Psychiatry, 172, 460-465.

[2] Tatsumi, H., Nakaaki, S., Torii, K., Shinagawa, Y., Watanabe, N., Murata, Y., Sato, J., Mimura, M. and Furukawa, T. (2009) Neuropsychiatric Symptoms Predict Change in Quality of Life of Alzheimer Disease Patients: A Two-Year Follow-Up Study. Psychiatry and Clinical Neurosciences, 63, 374-384. https://doi.org/10.1111/j.1440-1819.2009.01955.x

[3] Kristiina, K., Pertti, K., Asta, H., Matti, V., Tarja, V., Janne, M., Hannu, V., Juhani, S., Hilkka, S., Sirpa, H., Jaana, S. and Tuula, P. (2011) Neuropsychiatric Symptoms and Quality of Life in Patients with Very Mild and Mild Alzheimer's Disease. International Journal of Geriatric Psychiatry, 26, 473-482. https://doi.org/10.1002/gps.2550

[4] Matsumoto, N., Ikeda, M. and Fukuhara, R. (2007) Caregiver Burden Associated with Behavioral and Psychological Symptoms of Dementia in Elderly People in the Local Community. Dementia and Geriatric Cognitive Disorders, 23, 219-224. https://doi.org/10.1159/000099472

[5] Melo, G., Maroco, J. and de Mendonça, A. (2011) Influence of Personality on Caregiver's Burden, Depression and Distress Related to the BPSD. International Journal of Geriatric Psychiatry, 26, 1275-1282. https://doi.org/10.1002/gps.2677

[6] Ministry of Health, Labour and Welfare of Japan (2015) Guidelines for the Use of Psychotropic Drugs for BPSD Management for Family Doctors. http://www.mhlw.go.jp/stf/houdou/2r98520000036k0c.html

[7] Sarah, N.H., Andrew, J., Mc, L., David, G. and Le, C. (2007) Clinical Pharmacology in the Geriatric Patient. Fundamental \& Clinical Pharmacology, 21, 217-230. 
https://doi.org/10.1111/j.1472-8206.2007.00473.x

[8] Morimoto, T., Sakuma, M., Matsui, K., Kuramoto, N., Toshiro, J., Murakami, J., Fukui, T., Saito, M., Hiraide, A. and Bates, D.W. (2011) Incidence of Adverse Drug Events and Medication Errors in Japan: The JADE Study. Journal of General Internal Medicine, 26, 148-153. https://doi.org/10.1007/s11606-010-1518-3

[9] Chinen, S., Kanetake, N. and Fukuhara, A. (2012) Study of the Effect of Aromatherapy on Activities of Daily Life, Cognitive Function, and Behavioral and Psychological Symptoms among Elderly People with Dementia. Ryukyu Medical Journal, 31, 41-49.

[10] Okada, M. and Toki, H. (2010) Efforts to Reduce Behavioral Disorders in Patients with Dementia. Journal of the Kagawa Prefectural Nursing Society, 1, 1-3.

[11] Sakuraba, Y., Sasaki, S. and Ogiwara, M. (2016) Efforts to Reduce Wandering by Hand Massage for Dementia Patients. Journal of Japanese Society of Psychiatric Nursing, 59, 130-131.

[12] Sato, E., Muraoka, K. and Chichida, E. (2017) Improvement Effect of Peripheral Symptoms of Dementia by Introducing Hand Therapy to Elderly Patients with Dementia. Fukushima Prefectural Rural Medical Association Magazine, 57, $27-29$.

[13] Takahashi, S., Kobayashi, T. and Ogiwara, M. (2016) An Approach to Reduction in BPSD by Hand Massage in Patients with Dementia. Annual Meeting of the Japanese Psychiatric Nursing, 14, 90.

[14] Kolcaba, K., Dowd, T., Steiner, R. and Mitzel, A. (2004) Efficacy of Hand Massage for Enhancing the Comfort of Hospice Patients. Journal of Hospice \& Palliative Nursing, 6, 91-102. https://doi.org/10.1097/00129191-200404000-00012

[15] Kolcaba, K., Schirm, V. and Steiner, R. (2006) Effects of Hand Massage on Comfort of Nursing Home Residents. Geriatric Nursing, 27, 85-91. https://doi.org/10.1016/j.gerinurse.2006.02.006

[16] Kim, M.S., Cho, K.S., Woo, H.M. and Kim, J.H. (2001) Effects of Hand Massage on Anxiety in Cataract Surgery Using Local Anesthesia. Journal of Cataract \& Refractive Surgery, 27, 884-890. https://doi.org/10.1016/S0886-3350(00)00730-6

[17] Sato, T. (2006) Effects of Hand Massage on the Human Autonomic Nervous System and Mood in Healthy Women. Yamanashi Nursing Journal, 4, 25-32.

[18] Dodo, N. and Sakano, Y. (2009) Examination of Effectiveness of Relaxation to Control Anxiety for Patients with Alzheimer's Dementia. Behavioral Medicine Research, 15, 10-21.

[19] Mădălina, B., Géraldine, M., Caroline, A., Cécile, M. and Céline, G. (2015) Evaluation of the Preliminary Effectiveness of Hand Massage Therapy on Postoperative Pain of Adults in the Intensive Care Unit after Cardiac Surgery: A Pilot Randomized Controlled Trial. Pain Management Nursing, 16, 354-366. https://doi.org/10.1016/j.pmn.2014.08.014

[20] Tanaka, K. and Ogiso, K. (2010) Consideration of Alternative Therapies for Cancer Pain in Elderly Patients in the Terminal Stage; Hand Massage. Proceedings of the Japanese Nursing Association Geriatric Nursing, 40, 141-143.

[21] Kunikata, H., Watanabe, K., Miyosi, M. and Tanioka, T. (2012) The Effects Measurement of Hand Massage by the Autonomic Activity and Psychological Indicators. The Journal of Medical Investigation, 59, 206-212.

[22] Matsusita, M. and Morisita, T. (2003) A Study on Physiological Alteration and Psychological Evaluation through Purposeful Touch. Journal of Mie Prefectural College of Nursing, 7, 13-19. 
[23] Zhang, J., Dean, D., Nosco, D., Strathopulos, D. and Floros, M. (2006) Effect of Chiropractic Care on Heart Rate Variability and Pain in a Multisite Clinical Study. Journal of Manipulative and Physiological Therapeutics, 29, 267-274. https://doi.org/10.1016/j.jmpt.2006.03.010

[24] Ohta, Y., Deguchi, K., Sato, K., Matsuzono, K., Nakano, Y., Ikeda, Y., Wakutani, Y. and Takao, Y. (2015) A New Simple Score (ABS) for Assessing Behavioral and Psychological Symptoms of Dementia. Journal of the Neurological Sciences, 350, 14-17. https://doi.org/10.1016/j.jns.2015.01.029

[25] Suzuki, M. (2014) Hand Therapy Useful in Caring for Dementia. Ikeda Publishing, Tokyo. https://www.ikedashoten.co.jp/book-details.php?isbn=978-4-262-12357-8

[26] Margenfeld, F., Klocke, C. and Joos, S. (2019) Manual Massage for Persons Living with Dementia: A Systematic Review and Meta-Analysis. International Journal of Nursing Studies, 96, 132-142. https://doi.org/10.1016/j.ijnurstu.2018.12.012

[27] Ikeda, M. (2017) Behavioral and Psychological Symptoms of Dementia (BPSD). Neurotherapy, 34, 386. 